\title{
Image-enhanced modelling of residual compressive after impact strength in laminated composites
}

\author{
D. J. Bull ${ }^{1}$, S. M. Spearing ${ }^{1}$ and I. Sinclair ${ }^{1}$ \\ ${ }^{1}$ Faculty of Engineering and the Environment, University of Southampton, \\ Southampton, SO17 1BJ, United Kingdom \\ Email: daniel.bull@soton.ac.uk
}

Keywords: Buckling; Finite element analysis (FEA); Computed tomography (CT) analysis; virtual crack closure technique

This study implements key mechanisms and parameters observed from the previous compression after impact (CAI) experimental studies into finite element models, to study their effects parametrically on residual compression strength. The mechanisms and parameters include: impact damage area, the role of intact interfaces within the overall damaged region (identified here as the "undamaged cone"), interlaminar toughness, and the extent of permanent out-of-plane deformation. The findings highlight that within the limits observed experimentally, in addition to the size of the damaged area, interlaminar toughness and the extent of permanent out-of-plane deformation strongly affect CAI strength (by up to $100 \%$ for a given damage area), with toughness playing a more significant influence than permanent out-of-plane deformation. For the first time, and contrary to much of the literature, the significant role of the undamaged cone is evidenced and quantified.

\section{Introduction}

It is widely known that laminated carbon fibre reinforced polymers (CFRPs) are susceptible to low velocity impact damage, which directly affects compression after impact (CAI) strength. Many experimental studies report a correlation between the reduction in CAI strength and the impact damage area, attributing this loss of strength primarily to the extent of the delaminations [1-6].

Whilst delaminations are evidently a key contribution to loss of CAl strength, the sequence of events leading to compressive failure is the subject of ongoing debate within the literature. Whilst most studies report that local buckling results in a rapid growth of delaminations that extend laterally from the impact site, leading to a sudden loss of load carrying capability [7-9], others report a load redistribution resulting in compressive failure and kink band formation in material adjacent to the impact site [10-13].

Numerous modelling studies are reported in the literature. For simplicity, many studies represent the complex network of delaminations and intralaminar damage that occurs during impact into simple circular or elliptical delaminations [14-16]. More complex models simulate the impact damage itself, using this information to predict CAI behaviour [17]. Models can be purely elastic [18] or can include delamination growth, typically through use of cohesive zone modelling $[14,15,17]$ and/or continuum damage mechanics-based failure criteria (e.g. Hashin's) [15, 19]. Models have been constructed to include realistic damage patterns and parametrically compare different damage shapes to CAI failure load $[18,20]$. Whilst it is technically valuable to predict CAI processes to high accuracy, simplifications are of course desirable to ameliorate the computational cost. To optimise accuracy and computational cost, it is important to include the key mechanisms and associated chronology that contributes to CAl failure [21]. 
In previous experimental work carried out by the authors [22], micro-focus computed tomography $(\mu \mathrm{CT})$ was used to image damage within impacted coupons, followed by $\mu \mathrm{CT}$ scans taken from interrupted CAI tests immediately prior to failure. Such imaging has allowed unprecedented observation and quantification of the damage resulting from impact and its subsequent development in the CAI tests. An area of interest referred to as an "undamaged cone" has been the presence of a characteristic region of relatively undamaged material through the thickness of the specimen immediately under the impact point [22-25]. Delamination growth through the undamaged cone during CAl loading was shown to play a role in the CAl failure process by effectively connecting and extending the surrounding pattern of delaminations, prior to critical instability [22]. Differences in this behaviour were however identified and was attributed to different material systems and corresponding changes in interlaminar toughness. As such, modelling and optimising the CAI responses of different materials would appear to require proper consideration of behaviour within the undamaged cone.

Previous work also uncovered variations in residual CAl strength of up to $30 \%$ for a given total damage area, between material systems that varied in interlaminar toughness, see Figure 1 [22]. This plot highlights differences between the untoughened (UT) and several particle-toughened material systems (T1-T4) with the toughened systems showing greater retention of compression strength for a given size of damage (c.f. the untoughened systems). The variation in failure stress for a given damage area indicates that material toughness still plays a role in damage tolerance after an impact event. Additional factors affecting residual CAI strength have also been shown to extend to the laminate thickness where for a given damage size, variations in failure strain arise due to the formation of other damage modes such as fibre fracture [21]. It is therefore important that mechanisms beyond the size of the damage area are considered regarding modelling CAI failure.

Through use of finite element models, the present work aims to improve understanding of the contribution of three aspects (the undamaged cone, interlaminar toughness, and permanent out-ofplane deformation) to CAI performance, aiming to clarify which mechanisms of failure (delamination growth or $0^{\circ}$ compressive fibre fracture) contribute to catastrophic CAl failure. The intention of the work is to inform future experiments, improve modelling strategies, and to guide material development.

\section{Methodology}

\subsection{Setup of model}

Finite element modelling was conducted using ABAQUS ${ }^{\mathrm{TM}} 6.13$ explicit software. The model utilised a standard ASTM D7137 [26] test coupon geometry and boundary conditions. This comprised of a test coupon size of $150 \times 100 \times 4.8 \mathrm{~mm}$ using 24 plies and a [45/0/-45/90 $]_{3 S}$ stacking sequence. An IM7/8551-7 unidirectional carbon fibre system has been modelled as a 'baseline'; with elastic properties as shown in Table 1 [27]. To provide parametric variations of interlaminar toughness, five toughness groups were chosen ranging from systems with very low to high toughness compared to the experimentally determined value as shown in Table 2 . These represent a range of toughness values based on the authors' previous work [22-24, 28].

To model delamination growth, virtual crack closure technique (VCCT) elements were included in the model. Delamination occurs between the VCCT elements when the mixed mode criterion (Equation 1 ) is satisfied $[29,30] . G_{I}, G_{I I}, G_{I I I}$ represent the mode I, mode II and mode III components of strain 
energy release rate respectively and $G_{I C}, G_{I I C}, G_{I I I C}$ represent the critical strain energy release rates for their respective modes. In all models, the exponents $\alpha, \beta$ and $\chi$ were set to unity.

$$
\left(\frac{G_{I}}{G_{I C}}\right)^{\alpha}+\left(\frac{G_{I I}}{G_{I I C}}\right)^{\beta}+\left(\frac{G_{I I I}}{G_{I I I C}}\right)^{\chi} \geq 1
$$

\begin{tabular}{ccc}
\hline Property & & Value \\
\hline$E_{11}$ & {$[\mathrm{GPa}]$} & 165 \\
$E_{22}$ & {$[\mathrm{GPa}]$} & 8.4 \\
$E_{33}$ & {$[\mathrm{GPa}]$} & 8.4 \\
$G_{12}$ & {$[\mathrm{GPa}]$} & 5.4 \\
$G_{13}$ & {$[\mathrm{GPa}]$} & 5.4 \\
$G_{23}$ & {$[\mathrm{GPa}]$} & 2.8 \\
$v_{12}$ & - & 0.34 \\
$v_{13}$ & - & 0.34 \\
$v_{23}$ & - & 0.5 \\
\hline
\end{tabular}

Table 1: Material elastic properties.

\begin{tabular}{cccc}
\hline Toughness & $\begin{array}{c}\text { Mode } \\
\text { I } \\
\left(\mathrm{J} / \mathrm{m}^{2}\right)\end{array}$ & $\begin{array}{c}\text { Mode } \\
\text { II } \\
\left(\mathrm{J} / \mathrm{m}^{2}\right)\end{array}$ & $\begin{array}{c}\text { Mode III } \\
\left(\mathrm{J} / \mathrm{m}^{2}\right)\end{array}$ \\
\hline Very-low & 270 & 600 & 600 \\
Low & 394 & 875 & 875 \\
Medium & 765 & 1700 & 1700 \\
High & 1170 & 2600 & 2600 \\
Table 2: Material interlaminar toughness properties.
\end{tabular}

Figure 2 shows a schematic of the model setup, and how the permanent deformation representing the indent was added. To form the layup of the model, six stacked sublaminates each comprised of four plies, [45/0/-45/90] and [90/-45/0/45] for the sublaminates above and below the mid-plane respectively, were constructed from four-noded doubly curved general-purpose shells with reduced integration (S4R). The sublaminates were assembled using "tie" constraints enabling pairs of surfaces between sublaminates to be joined together. Delaminations were included between sublaminate pairs and were formed of circular areas. The shape was chosen to consolidate the "spiral staircase" delamination pattern observed experimentally $[22,25]$ into a single circular delamination to minimize the complexity and solution time of the model. Additionally, fibre fracture and matrix cracks where omitted from the model to maintain simplicity. Delaminations between pairs of sublaminates were formed of untied regions and used contact behaviour to prevent surface penetration. The contact surface encompassed a frictionless and hard contact property. To model delamination growth, virtual crack closure technique (VCCT) elements were included in the undamaged cone region and along the perimeter surrounding the outer delaminated region. The undamaged cone in the current models was set to $5 \mathrm{~mm}$ in diameter in all cases, in keeping with previous experimental observations[22].

To enable local buckling in the model, an imperfection was added to the centre of the sublaminates by displacing the respective nodes out-of-plane, i.e. representing residual permanent deformation from an impact event. The indented surface was modelled as a spherical cap with a curvature 
matching the radius $(R=94 \mathrm{~mm})$ observed in experimental work [22] and was simply applied so that all the sublaminates had the same shape. The peak depth $(\mathrm{h})$ of the imperfection ranged from 0.02 to $0.4 \mathrm{~mm}$.

Boundary conditions were set to replicate the constraints outlined in the ASTM D7137 CAI tests. An in-plane compressive edge displacement of $1 \mathrm{~mm}$ was applied to the top edges of the shells to occur over half a second duration

To enable a reasonable solution time, a time step of 73 ns was used with fixed mass scaling. The kinetic energy for each test was monitored and remained two orders of magnitude below the recommended $10 \%$ of total internal energy. Models were solved on the University of Southampton Iridis 4 supercomputer cluster. Each model was solved using 64 cores over 4 nodes with a total solution time in the range of $30-34$ hours.

In this work, the critical load carrying capability was calculated when either the $0^{\circ}$ compressive stress exceeded the corresponding compressive strength (Xc) of $1.6 \mathrm{GPa}$, or at the onset of delamination growth at the lateral edge of the damage area (see section 3.1).

\subsection{Parametric study}

For the full parametric study, all combinations of the parameters shown in table 3 were used, giving 96 cases analysed.

\begin{tabular}{cccc}
\hline $\begin{array}{c}\text { Damage } \\
\text { diameter }(\mathrm{mm})\end{array}$ & Toughness & $\begin{array}{c}\text { Permanent out-of-plane } \\
\text { deformation }(\mathrm{mm})\end{array}$ & Undamaged cone \\
\hline 20 & V-low & 0.02 & $\begin{array}{c}\text { No undamaged cone } \\
\text { With undamaged } \\
30\end{array}$ \\
Low & 0.04 & cone \\
40 & Medium & 0.1 & \\
& High & 0.4 &
\end{tabular}

Table 3: Test matrix parameters.

\section{Results}

\subsection{CAl failure mechanisms and failure criterion}

At the onset of CAI failure, a compressive stress concentration is identifiable at the outer edges of the impact delamination (Figure 3, annotated as (a)). These stresses may then exceed the local compressive strength, $\mathrm{Xc}$, leading to a loss of local load carrying capability. Alternatively, postbuckling can lead to rapid delamination propagation at the lateral edges of the damage area. In the example shown in Figure 4, delamination growth is observed into the undamaged cone (c) followed by rapid delamination growth extending laterally outwards (d). For the modelling carried out here, the failure chronology is seen to change with prior damage area, toughness and extent of permanent out-of-plane deformation, as expanded upon in the following sections

\subsection{Effect of the undamaged cone}

Figure 5 shows the effects of the presence of an undamaged cone on the buckling behaviour for 20, 30 , and $40 \mathrm{~mm}$ delamination diameters. The out-of-plane displacement was recorded at the central node. Systems containing the undamaged cone exhibited a clear reduction in out-of-plane displacement for a given load. This difference was shown to be up to $50 \%$ and was consistent at all damage diameters and all permanent out-of-plane deformations tested. 
Figure 6 shows how the inclusion of the undamaged cone affects the three modes of normalised peak strain energy release rate measured at the outer perimeter of the delamination area. The strain energy release rate was normalised against the "high toughness" (see Table 2) critical energy release rate values. The undamaged cone is shown in all cases to reduce the peak components of mode I and mode III strain energy release rates and in most cases the mode II component. Figure 6 also shows the relative contributions of the fracture modes to the total strain energy release rate with mode II being the largest followed by mode III.

Figure 7 highlights the importance of the undamaged cone for the peak $0^{\circ}$ compressive stress. The plot shows the peak compressive stress within the test coupon for a given applied load. At the point where the critical stress, $\mathrm{Xc}$, is reached at $1.6 \mathrm{GPa}$, there is a $30 \%$ difference in the applied load between the model containing the undamaged cone and that without the undamaged cone.

There is a link between the out-of-plane displacement (Figure 5), increase in strain energy release rate (Figure 6) and peak compressive stress (Figure 7). In the post-buckled regime where the rate of out-of-plane displacement increases, there is a corresponding accelerated increase in peak strain energy release rate and $0^{\circ}$ compressive stress.

The importance of the undamaged cone mechanism is clear in Figure 8 where its presence is shown to lead consistently to an increase in predicted failure load across all damage areas and prior out-ofplane deformation, by up to $80 \%$.

\subsection{Effect of toughness}

Delamination growth into the undamaged cone has been observed in some systems prior to critical loss of load carrying capability, which may then play a key role in controlling failure [22]. In the plot shown in Figure 9, at $140 \mathrm{kN}$ there is divergence of the load vs. peak compressive stress between the various toughened systems. The low, and very low, toughness cases exhibited delamination growth into the undamaged cone, whereas the higher toughness cases did not. The loss of traction between sublaminates in the undamaged cone region for the lower toughness cases reduced the local bending stiffness, leading to a rapid increase in out-of-plane displacement and early increase in peak compressive stresses at the outer edge of the delamination. In the medium and high toughness systems, very little damage growth was observed into the undamaged cone, resulting in a similar overall response to the infinite toughness case. Correspondingly, toughness is shown to play a substantial role in determining the overall failure load. Figure 10 shows the failure loads of systems with initial damage of 20,30 and $40 \mathrm{~mm}$ diameters across the four toughened systems. The variation in toughness results in a range of failure loads, with more than a $50 \%$ difference particularly identified at 30 and $40 \mathrm{~mm}$ delamination diameters. The magnitude of failure load variations for a given damage diameter/area is in agreement with previously published experimental work by the authors, see Figure 1 [22].

The data points circled in Figure 10 represent failure governed by the stress exceeding the critical $0^{\circ}$ compressive strength, whereas the other data points reflect failure occurring by rapid delamination growth, highlighting the competition between failure mechanisms. Nonetheless, in both instances toughness plays a significant role in determining failure load, either through supressing delamination growth into the undamaged cone or by suppressing delamination growth at the lateral edges of the delaminated area.

\subsection{Effect of permanent out-of-plane deformation}

The effects of permanent out-of-plane deformation at the impact point were tested against the failure load at 20, 30 and $40 \mathrm{~mm}$ damage diameters, see Figure 11 . At 20 and $30 \mathrm{~mm}$ damage diameters, there is very little difference $(<5 \%)$ in failure load across the range of permanent out-of- 
plane deformation levels. The range of failure loads however is shown to increase to $\sim 25 \%$ difference at $40 \mathrm{~mm}$ damage diameter where this behaviour is most significant.

Interestingly the systems with the largest permanent out-of-plane deformations exhibited a greater failure load at $40 \mathrm{~mm}$ damage diameter. This effect is associated with the post-buckling response shown in Figure 12. In each of the permanent out-of-plane deformations there is a bifurcation "knee" point corresponding to buckling followed by a rapid increase in out-of-plane deflection in the postbuckle regime. The severity of the knee point and the divergence between the pre- and post-buckled responses decreases with an increase in the initial permanent out-of-plane deformation, consistent with the general effect of initial imperfections on structural stability. However, the rank order of the out-of-plane deflection for a given load is shown to reverse beyond the knee point where the lines are shown to cross; this is most significant for the larger initial permanent deformations of 0.1 and $0.4 \mathrm{~mm}$.

\section{Comparison with experimental studies: damage mechanisms and buckling behaviour}

Comparison between experimental and FE predictions are made to assess if mechanisms are captured accurately, particularly delamination growth and buckling behaviour. One limitation of this study is direct comparison of the predicted failure loads with experiments. Due to use of proprietary development materials, individual fracture toughness values of the experimental coupons are not available, making this comparison difficult. However, it is clear that both experimental (Figure 1 [22]) and FE predictions (Figure 10 and Figure 11) resulted in variations in failure load of the same order for a given damage size due to differences in material toughness.

\subsection{Delamination propagation}

Figure 13 shows a comparison between delamination growth for (a) FE and (b) experimental studies. The experimental study is described in previously published work, which used $\mu \mathrm{CT}$ scans to capture damage growth mechanisms in CAI tests [22]. Both images in the figure are at the same ply interface, i.e. the upper third and fourth ply interfaces in the experimental specimens which correspond to delaminations in the first and second sublaminate interface in the FE model. Delaminations are shown to propagate into the undamaged cone (i) with the dotted region representing the initial undamaged zone after impact. At the outer extremities of the delamination area, damage growth in this region (ii) was captured with both techniques. As such, the FE models appear to be consistent with the measured incidence of delamination growth at both locations.

\subsection{Buckling behaviour}

The buckling mode shape and scale are compared between experimental and FE predictions: Figure 14. (a) shows buckling behaviour captured experimentally using stereo digital image correlation, whilst (b) shows the buckling behaviour from the FE predictions. Both cases are of the same region showing buckling at the non-impacted side of the test coupon. Comparisons between FE and experimental results indicate reasonable agreement in the buckled mode shape, size, and aspect ratio. For clarity, the regions highlighted by the dotted regions in (a) were caused by two loose tows on the back surface and, as such, should be disregarded. The buckled shape of the test coupon agrees with other stereo digital image correlation work on carbon/epoxy coupons of the same geometry [31]. 


\section{Discussion}

The primary objective of this modelling work was to build on observations made using X-ray computed tomography, to understand the factors affecting the compression after impact response of composite laminates. The results are consistent with the assertion that the undamaged cone may indeed be important in determining the post impact failure load and is in agreement with another study where the undamaged cone approximately doubled the stress during buckling and postbuckling [18]. It has also evidenced a linked role of toughness in determining both the CAI strength as well as the damage area created by the impact event. The modelling indicates that there is a close competition between failure due to exceeding the compressive strength of the $0^{\circ}$ plies and lateral delamination growth. The distinction between these failure modes is difficult to establish experimentally. The modelling presented herein gives a reasonable indication that variations in CAI previously reported in the literature may be due to relatively small differences in the properties, particularly toughness and $0^{\circ}$ ply strength, across the range of materials investigated in these studies. Furthermore, the present work indicates that regardless of which of the failure modes is operating, the interlaminar toughness may play a role in determining damage tolerance (as measured by CAI for a given extent of prior damage), as well as in determining damage resistance (as quantified by the impact damage area).

It is recognised that a simplified damage pattern was used in this work: specifically, the consolidation of the 'spiral staircase' of delaminations into single circular delaminations is not truly representative. Increasing the number of shell elements to capture a more representative pattern of delaminations may be expected to lead to more accurate CAI predictions, but would inevitably lead to longer numerical solution times. Similarly, kink-band formation, fibre fracture and matrix cracking were not included in the model, but were observed experimentally [22]. As such, a comprehensive mechanistic analysis may be anticipated to elucidate further the potential coupling with the processes investigated here.

\section{Conclusions and closing remarks}

Simulations indicate that residual out-of-plane deformation, interlaminar toughness and the presence of an undamaged cone all play a role in residual compressive strength, in addition to the size of the prior impact damage area. The undamaged cone appears to play an important role by increasing the buckling load and post-buckled response. This in turn reduces the compressive stresses and magnitude of strain energy release rate at the outer edge of the damage area for a given applied load. To model accurately the effect of impact on the subsequent structural response it is therefore considered important to include such features, as variations of up to $80 \%$ in the failure load were predicted when systems with and without the undamaged cone were compared directly.

The work shows that tougher systems result in significantly higher CAl residual strength for a given damage area, with a 50\% difference in failure load being observed between the lowest and highest toughness systems in this study which included the undamaged cone. It is, therefore, clear that improvements particularly in the mode II and mode III toughness would be expected to improve the residual CAI strength, as well as reducing the damage area due to the initial impact event. The benefits of increased toughness were observed both in cases where delamination growth into the undamaged cone occurred, as well as where rapid delamination growth occurred at the outer edges of the damage area. The extent of permanent deformation due to the initial impact was shown to have less effect compared to variation in the toughness, resulting in just a $~ 5 \%$ difference in residual strength at 20 and $30 \mathrm{~mm}$ damage diameter, but was more significant at $40 \mathrm{~mm}$ damage diameter 
where a difference of $\sim 25 \%$ to the failure loads was observed. It is interesting to note that the systems which exhibited the greatest permanent out-of-plane deformation exhibit a higher failure load at $40 \mathrm{~mm}$ damage diameter. This is an important observation, as toughened systems, as reported in previously published experimental work, typically exhibit a greater extent of permanent out-of-plane deformation, associated with local inelasticity (pseudo-plasticity). The present results suggest that this feature of toughened systems does not override the benefit of higher toughness in making a positive contribution to the residual CAI load.

Overall, the use of finite element modelling informed by high resolution computed tomography observations has allowed novel insights to be gained into the competition between failure mechanisms and the relative importance of key material parameters in determining CAl response.

\section{Acknowledgements}

The authors are grateful for the support of Cytec Solvay Group with special thanks to Dr. Matthew Jackson. The authors are also appreciative of the EPSRC Impact Acceleration grant (EP/K503770/1) and the $\mu$-VIS X-ray Imaging Centre at the University of Southampton supported by EPSRC grant EPH01506X. The authors acknowledge the use of the IRIDIS High Performance Computing Facility, and associated support services at the University of Southampton, in the completion of this work.

\section{References}

[1] Cartié DDR, Irving PE. Effect of resin and fibre properties on impact and compression after impact performance of CFRP. Composites Part A: Applied Science and Manufacturing. 2002;33:483-93.

[2] Delfosse D, Poursartip A. Energy-based approach to impact damage in CFRP laminates. Composites Part a-Applied Science and Manufacturing. 1997;28:647-55.

[3] Kim N, Achenbach JD. Quantitative characterization of multiple delaminations in laminated composites using the compton backscatter technique. Journal of Nondestructive Evaluation. 1998;17:53-65.

[4] Recker HG, Altstadt V, Eberle W, Folda T, Gerth D, Heckmann W, et al. Toughened thermosets for damage tolerant carbon-fiber reinforced composites. Advanced Materials : The Big Payoff. 1989;21:283-93.

[5] Reis L, De Freitas M. Damage growth analysis of low velocity impacted composite panels. Composite structures. 1997;38:509-15.

[6] Rio TGD, Zaera R, Barbero E, Navarro C. Damage in CFRPs due to low velocity impact at low temperature. Composites Part B-Engineering. 2005;36:41-50.

[7] Chai $\mathrm{H}$, Knauss W, Babcock C. Observation of damage growth in compressively loaded laminates. Experimental Mechanics. 1983;23:329-37.

[8] Yan H, Oskay C, Krishnan A, Xu LR. Compression-after-impact response of woven fiber-reinforced composites. Composites Science and Technology. 2010;70:2128-36.

[9] de Freitas M, Reis L. Failure mechanisms on composite specimens subjected to compression after impact. Composite structures. 1998;42:365-73.

[10] Soutis C, Curtis P. Prediction of the post-impact compressive strength of CFRP laminated composites. Composites Science and Technology. 1996;56:677-84.

[11] Wisnom M. The role of delamination in failure of fibre-reinforced composites. Philosophical Transactions of the Royal Society A: Mathematical, Physical and Engineering Sciences.

2012;370:1850-70.

[12] Rivallant S, Bouvet C, Hongkarnjanakul N. Failure analysis of CFRP laminates subjected to compression after impact: FE simulation using discrete interface elements. Composites Part aApplied Science and Manufacturing. 2013;55:83-93. 
[13] Uda N, Ono K, Kunoo K. Compression fatigue failure of CFRP laminates with impact damage. Composites Science and Technology. 2009;69:2308-14.

[14] Suemasu H, Sasaki W, Ishikawa T, Aoki Y. A numerical study on compressive behavior of composite plates with multiple circular delaminations considering delamination propagation. Composites Science and Technology. 2008;68:2562-7.

[15] Abir MR, Tay TE, Ridha M, Lee HP. Modelling damage growth in composites subjected to impact and compression after impact. Composite structures. 2017;168:13-25.

[16] Esrail F, Kassapoglou C. An efficient approach to determine compression after impact strength of quasi-isotropic composite laminates. Composites Science and Technology. 2014;98:28-35.

[17] Tan W, Falzon BG, Chiu LNS, Price M. Predicting low velocity impact damage and CompressionAfter-Impact (CAI) behaviour of composite laminates. Composites Part a-Applied Science and Manufacturing. 2015;71:212-26.

[18] Craven R, lannucci L, Olsson R. Delamination buckling: A finite element study with realistic delamination shapes, multiple delaminations and fibre fracture cracks. Composites Part A: Applied Science and Manufacturing. 2010;41:684-92.

[19] Riccio A, Pietropaoli E. Modeling damage propagation in composite plates with embedded delamination under compressive load. Journal of composite materials. 2008;42:1309-35.

[20] Suemasu H, Sasaki W, Aoki Y, Ishikawa T. Compressive behavior of impact damaged composite laminates. In Proc 16th Int Conf on Composite Materials, Kyoto, Japan 8-13 July 20072007.

[21] Olsson R. Modelling of impact damage zones in composite laminates for strength after impact. Aeronautical Journal. 2012;116:1349-65.

[22] Bull DJ, Spearing SM, Sinclair I. Observations of damage development from compression-afterimpact experiments using ex situ micro-focus computed tomography. Composites Science and Technology. 2014;97:106-14.

[23] Bull D, Scott A, Spearing S, Sinclair I. The influence of toughening-particles in CFRPs on low velocity impact damage resistance performance. Composites Part A: Applied Science and Manufacturing. 2014;58:47-55.

[24] Bull D, Spearing S, Sinclair I, Helfen L. Three-dimensional assessment of low velocity impact damage in particle toughened composite laminates using micro-focus X-ray computed tomography and synchrotron radiation laminography. Composites Part A: Applied Science and Manufacturing.

2013;52:62-9.

[25] Bull DJ, Spearing SM, Sinclair I. Investigation of the response to low velocity impact and quasistatic indentation loading of particle-toughened carbon-fibre composite materials. Composites Part A: Applied Science and Manufacturing. 2015;74:38-46.

[26] Astm D7137: Standard Test Method for Compressive Residual Strength Properties of: Astm, 2012.

[27] Kaddour AS, Hinton MJ. Input data for test cases used in benchmarking triaxial failure theories of composites. Journal of composite materials. 2012;46:2295-312.

[28] Bull D, Helfen L, Sinclair I, Spearing S, Baumbach T. A comparison of multi-scale 3D X-ray tomographic inspection techniques for assessing carbon fibre composite impact damage.

Composites Science and Technology. 2013;75:55-61.

[29] Marat-Mendes RM, Freitas MM. Failure criteria for mixed mode delamination in glass fibre epoxy composites. Composite structures. 2010;92:2292-8.

[30] Reeder JR. An Evaluation of Mixed-Mode Delamination Failure Criteria. NASA Langley Technical Report Server; 1992.

[31] Sztefek P, Olsson R. Nonlinear compressive stiffness in impacted composite laminates determined by an inverse method. Composites Part a-Applied Science and Manufacturing. 2009;40:260-72. 


\section{Figures}

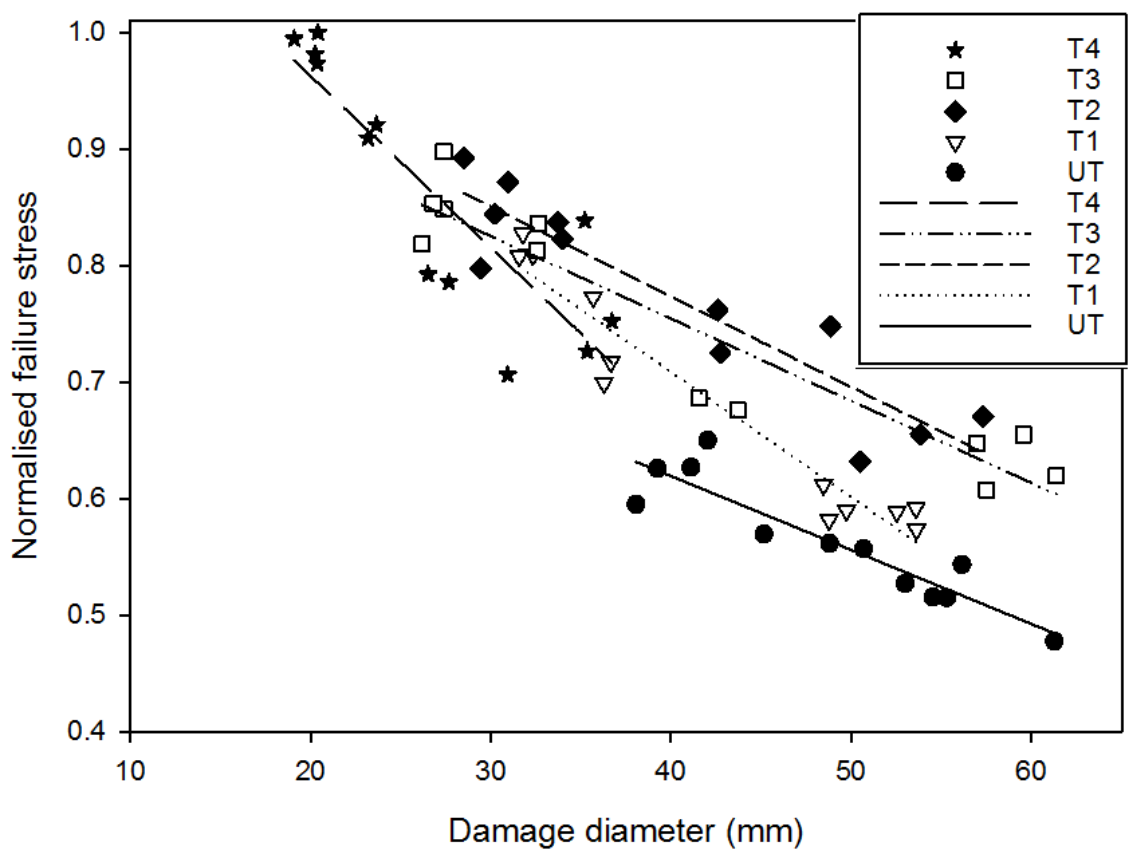

Figure 1: Plot of failure stress vs. impact damage diameter across five material system. The plot shows variations in failure stress after impact for a given damage size between one untoughened (UT) and four toughened (T1-T4) systems.

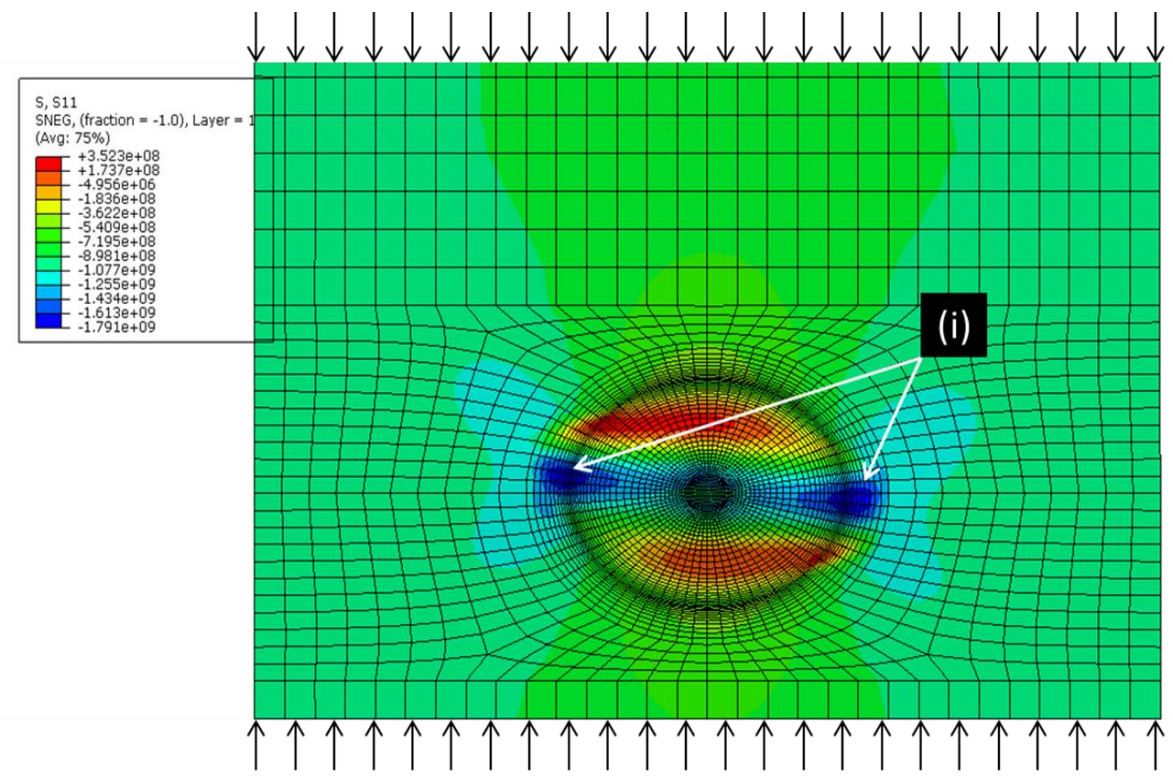

Figure 2: Schematic of model setup and permanent deformation. 


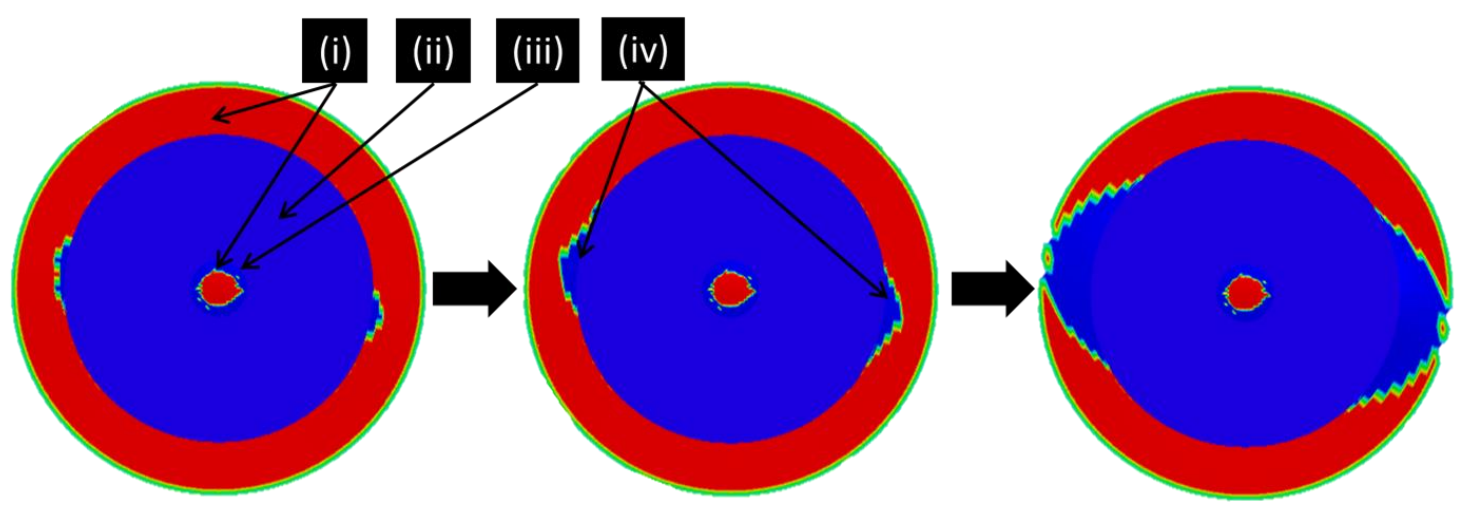

Figure 3: FE model showing $0^{\circ}$ in-plane compressive stresses. (i) local $0^{\circ}$ ply compressive stress concentration at outer edges of delamination in the post-buckled regime.

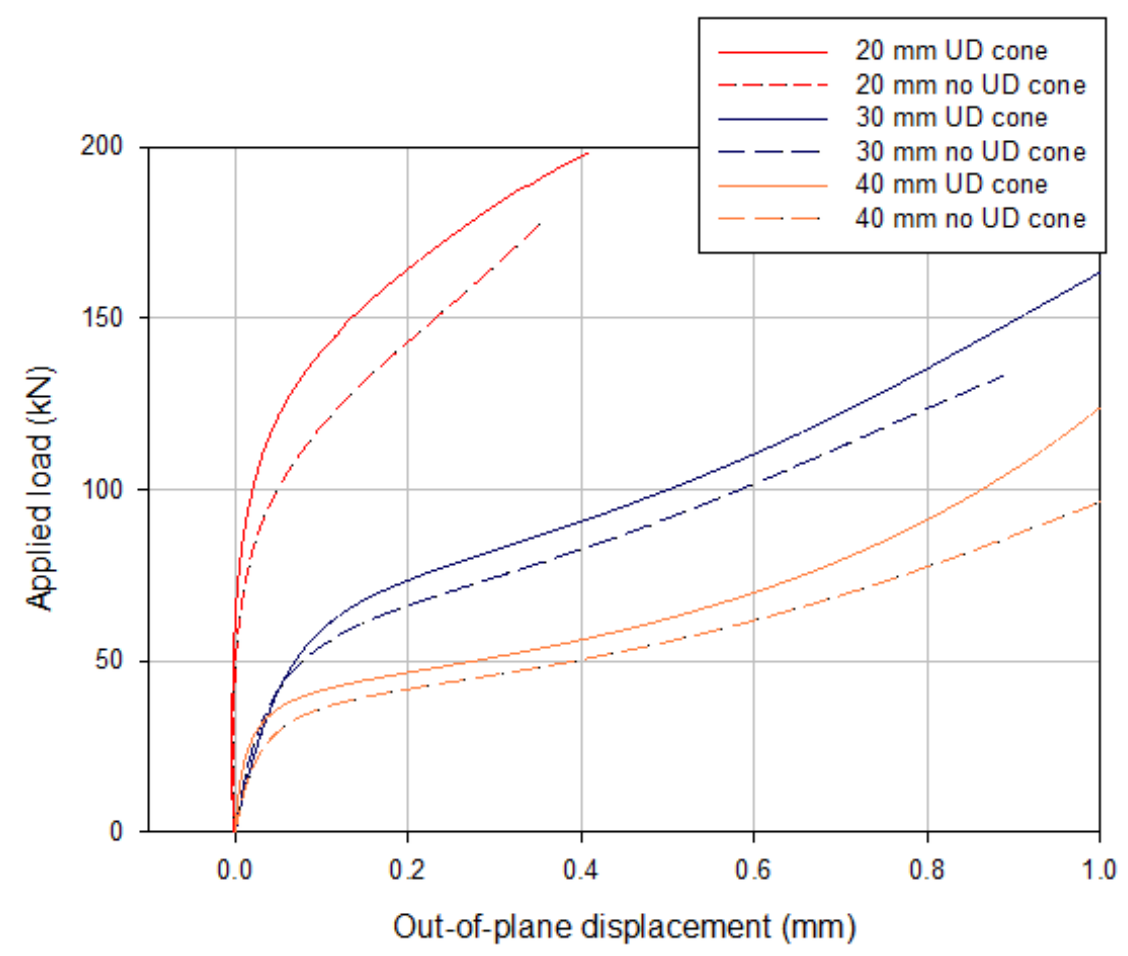

Figure 4: Simulated delamination growth behaviour, annotating specific growth processes: (a) at the undamaged cone and surrounding the delamination perimeter, (b) outer delamination region, (c) undamaged cone with partial delamination growth into this region, (d) rapid lateral delamination growth at the outer delamination edges. 


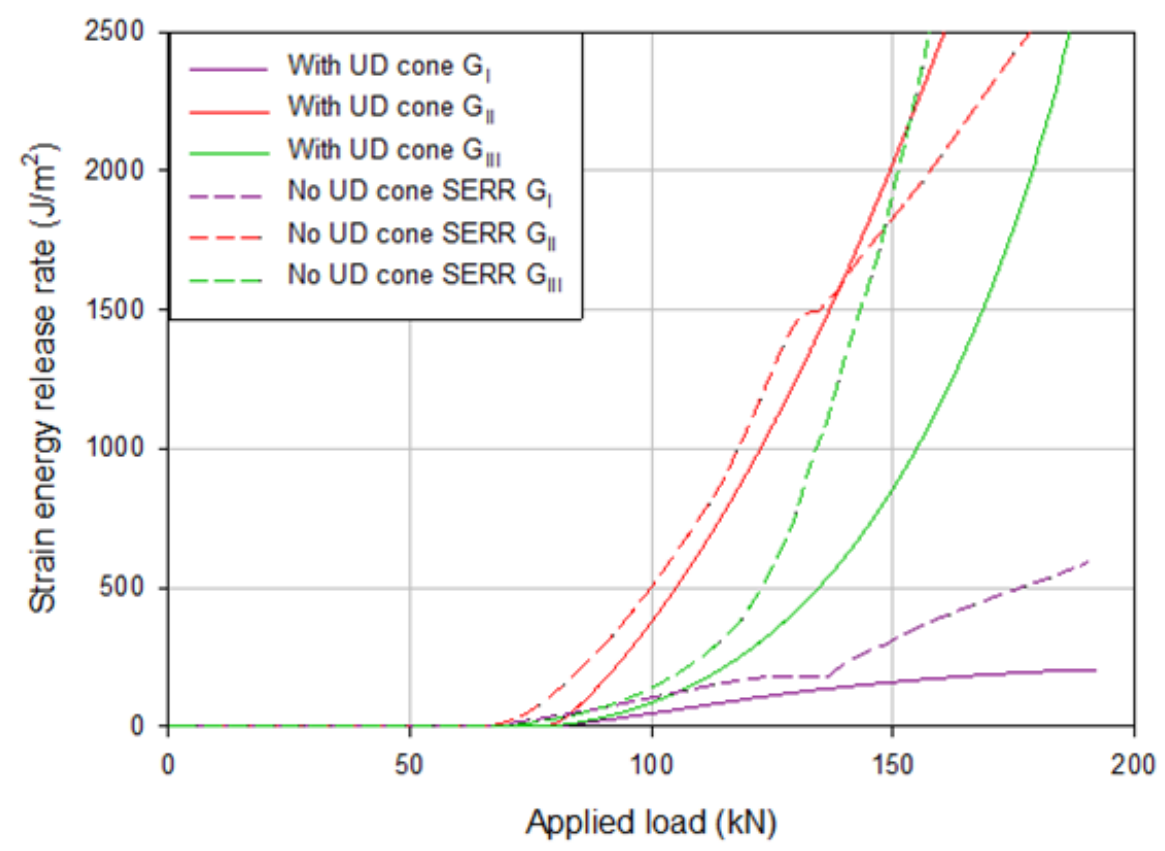

Figure 5: Role of the undamaged cone: on out-of-plane response without inclusion of delamination growth. Plots shown are taken at 20,30 and $40 \mathrm{~mm}$ delamination diameters, with (solid lines) and without (dashed lines) the undamaged cone at $0.1 \mathrm{~mm}$ permanent out-of-plane deformation.

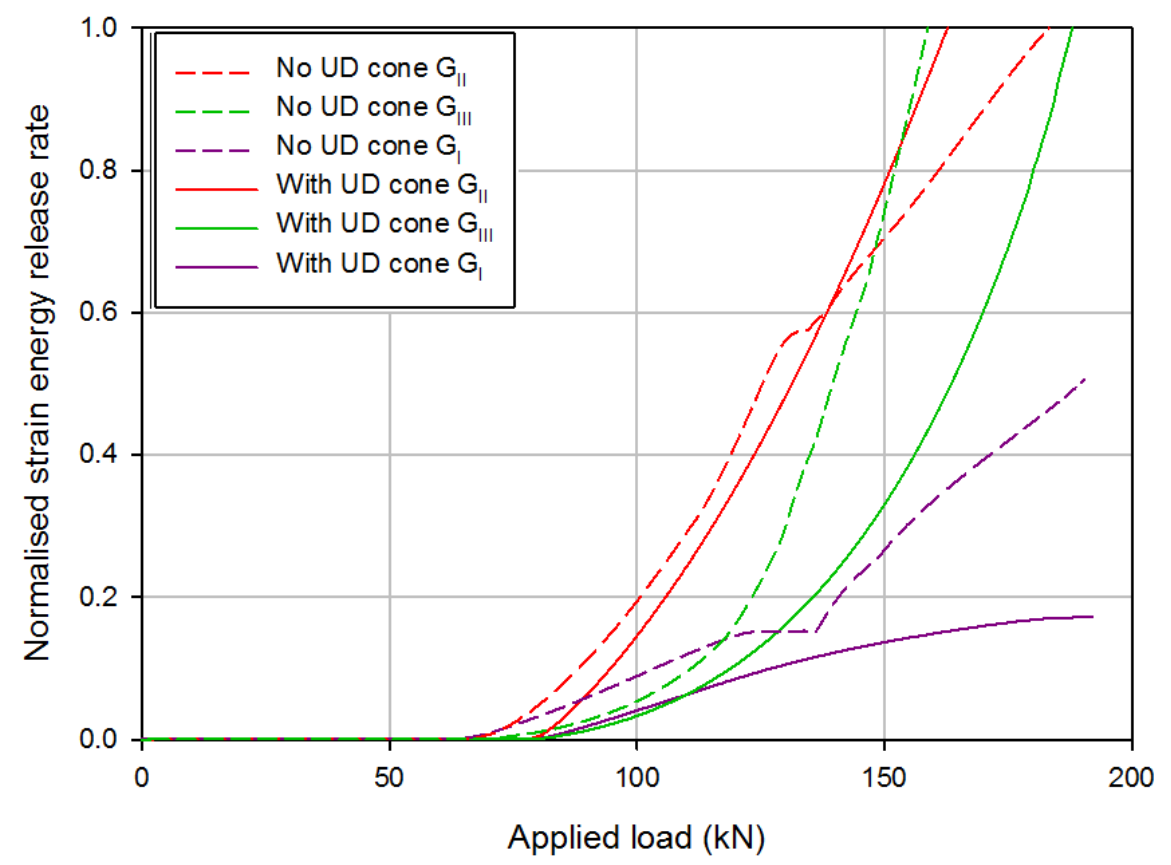

Figure 6: Role of the undamaged cone: a comparison between strain energy release rate and applied load. Plots shown are taken from $30 \mathrm{~mm}$ damage diameter with $0.04 \mathrm{~mm}$ permanent outof-plane deformation. 


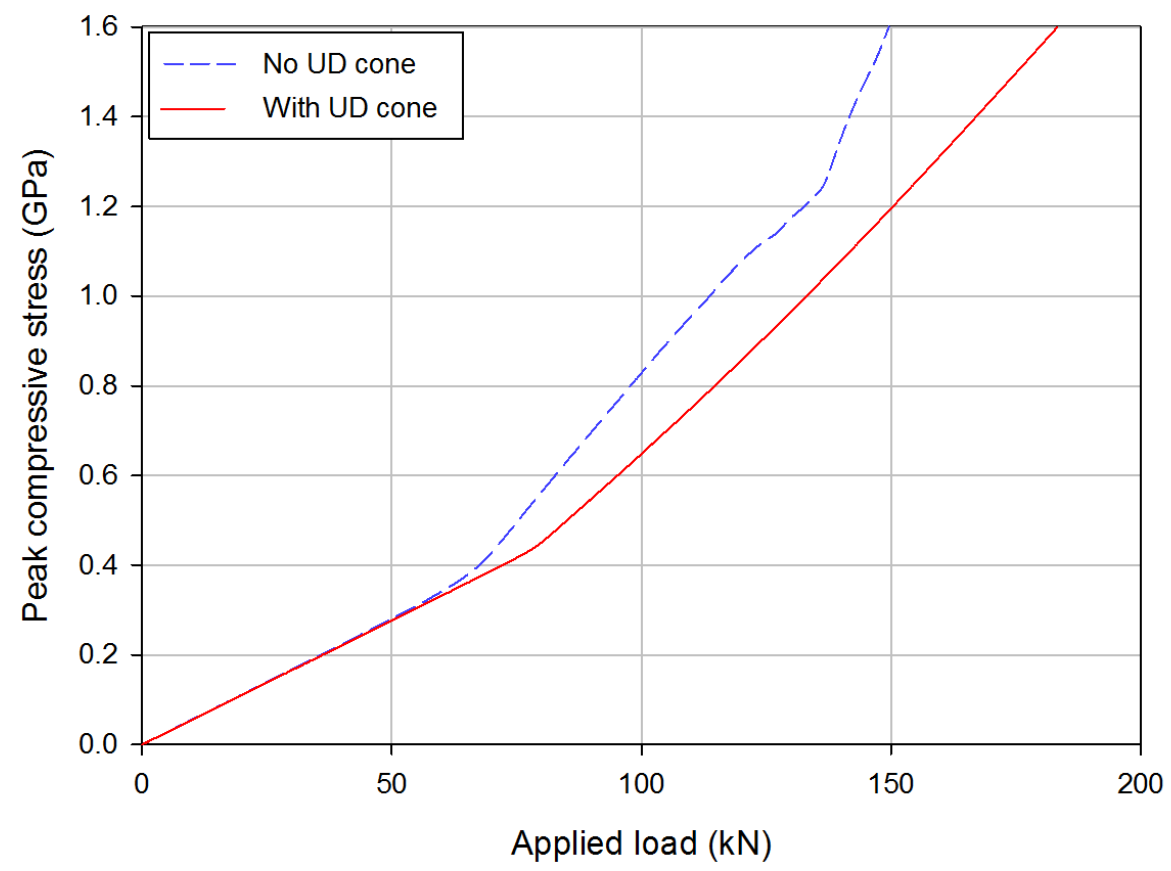

Figure 7: Role of the undamaged cone: a comparison between load and peak $0^{\circ}$ compressive stress. Plots shown are taken from $\mathbf{3 0} \mathrm{mm}$ damage diameter with $0.04 \mathrm{~mm}$ permanent out-of-plane deformation.

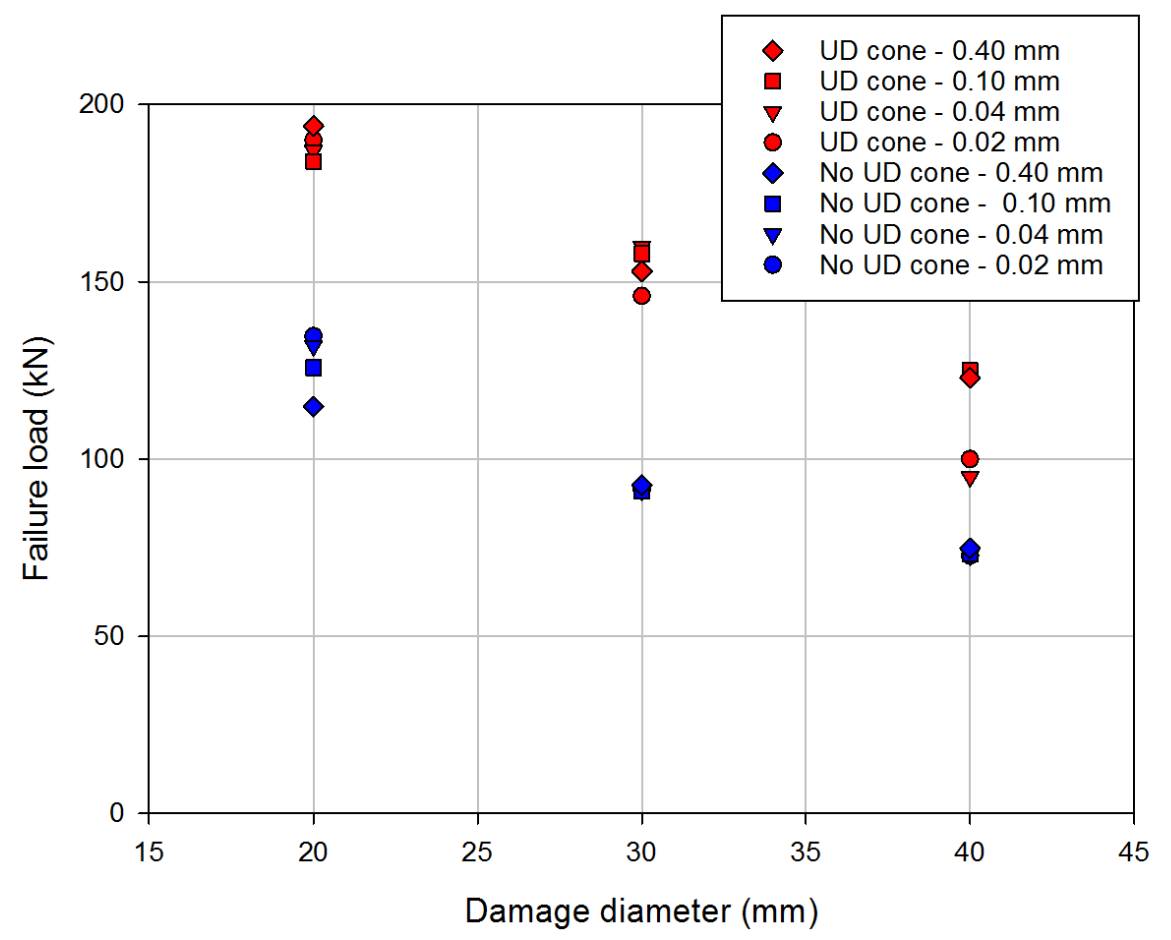

Figure 8: Comparison of failure loads for models with and without the undamaged (UD) cone at various magnitudes of out-of-plane permeant deformation. 


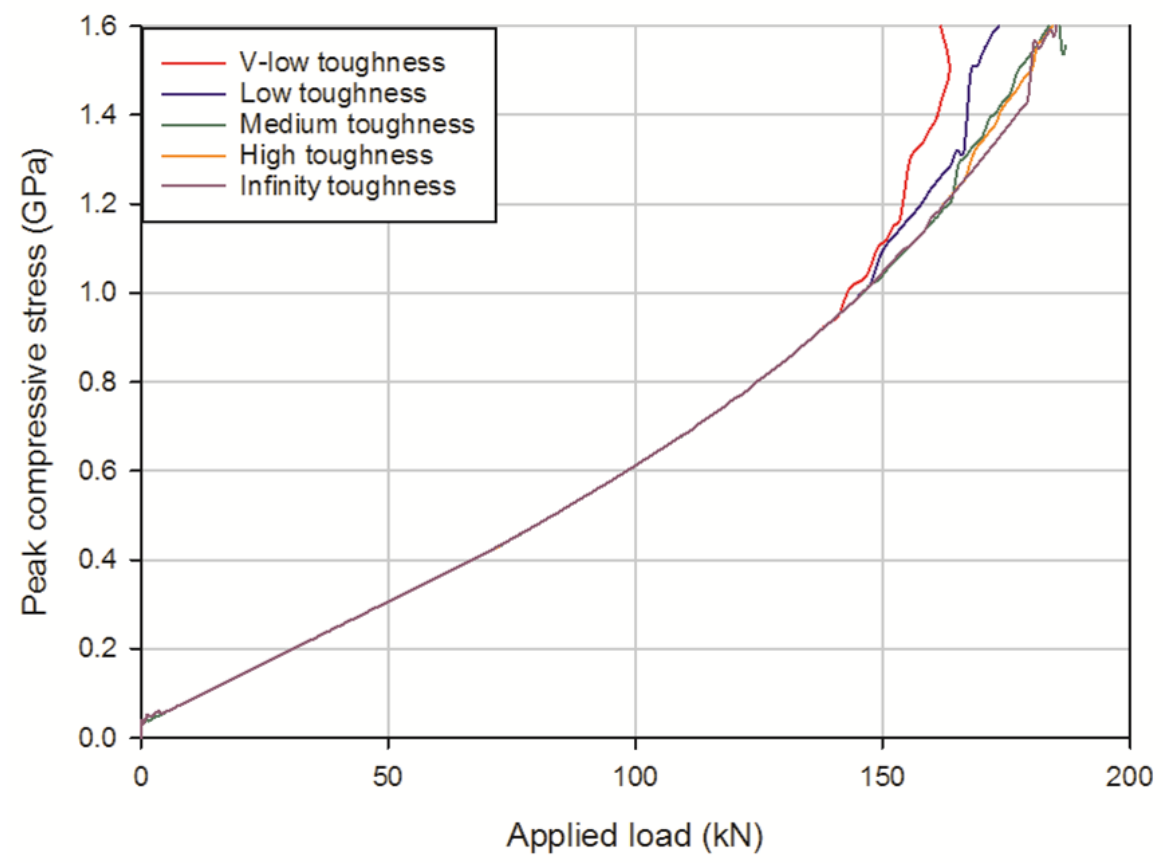

Figure 9: Effect of toughness on load vs. peak compressive stress. Plots shown are taken from 20 $\mathrm{mm}$ diameter damage area with $0.02 \mathrm{~mm}$ permanent out-of-plane deformation.

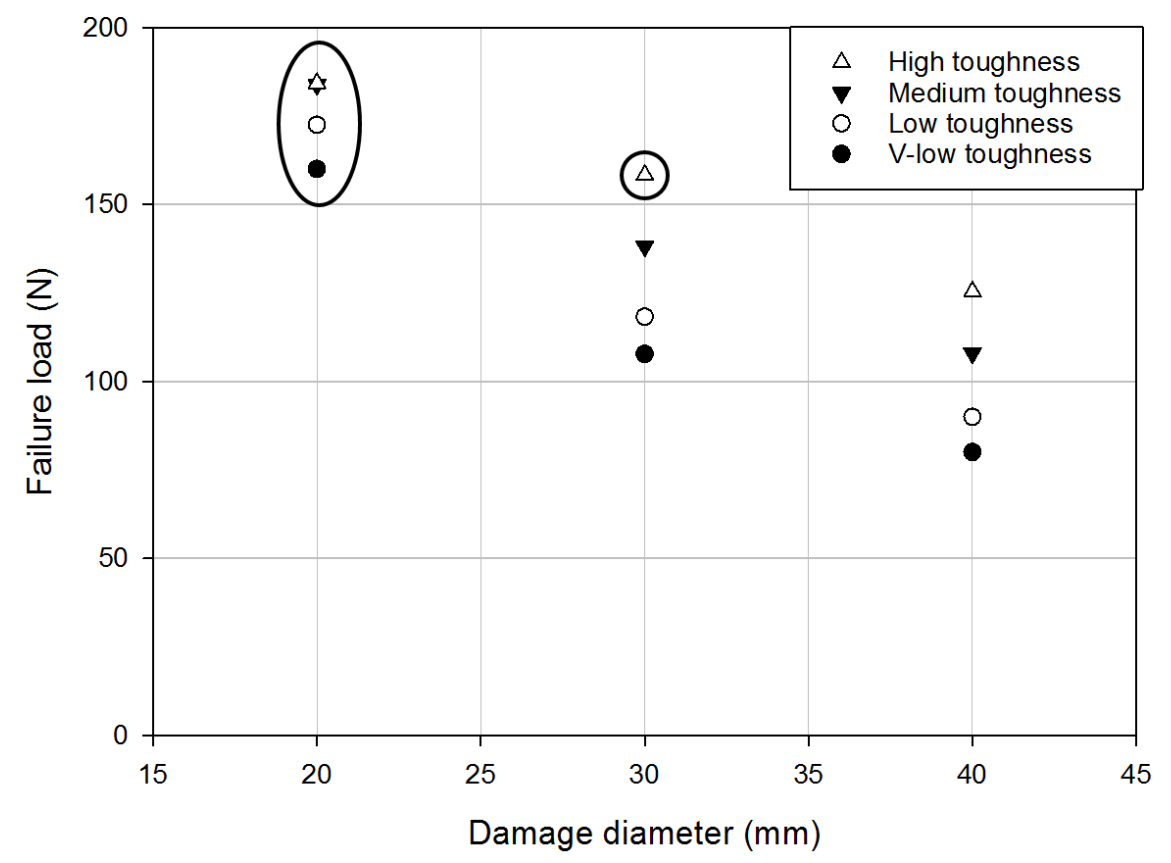

Figure 10: Effect of damage diameters and toughness on failure load. All points on the graph correspond to $0.1 \mathrm{~mm}$ permanent out-of-plane deformation. Circled points represent failure governed by the stress exceeding the critical $0^{\circ}$ compressive strength, uncircled data points represents failure occurring through rapid delamination growth. 


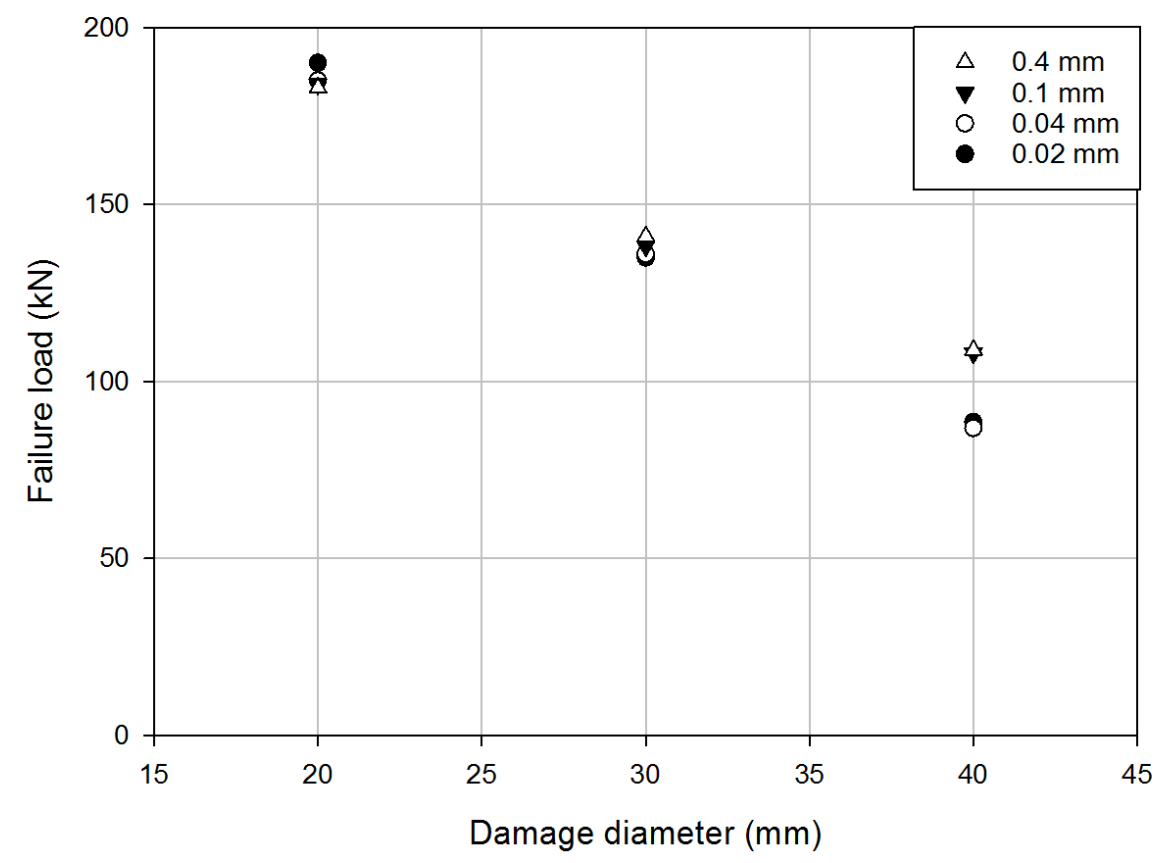

Figure 11: Effect of damage diameter and initial permanent out-of-plane deformation on failure load. All data points shown correspond to the medium toughness system.

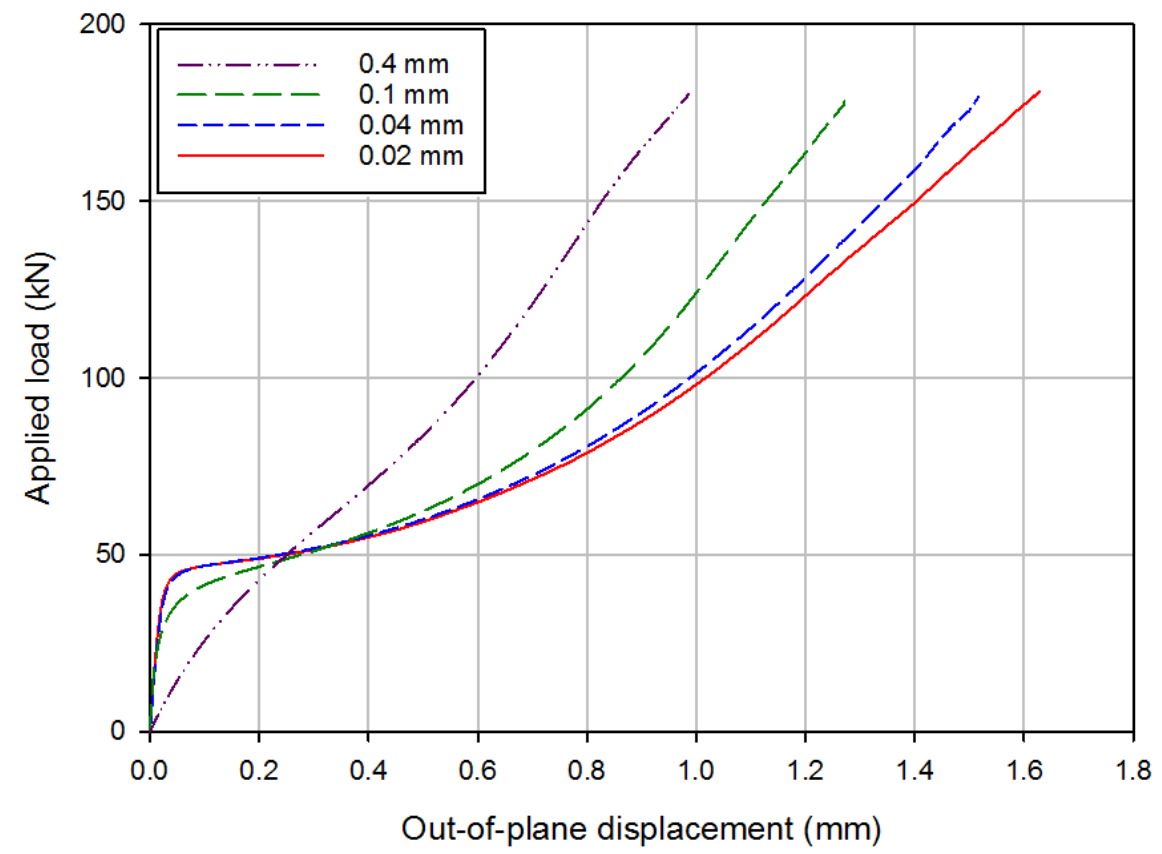

Figure 12: Effect of permanent out-of-plane deformation on load deflection response. All data points in the plot are taken from $\mathbf{4 0} \mathrm{mm}$ damage diameter models without damage growth. 


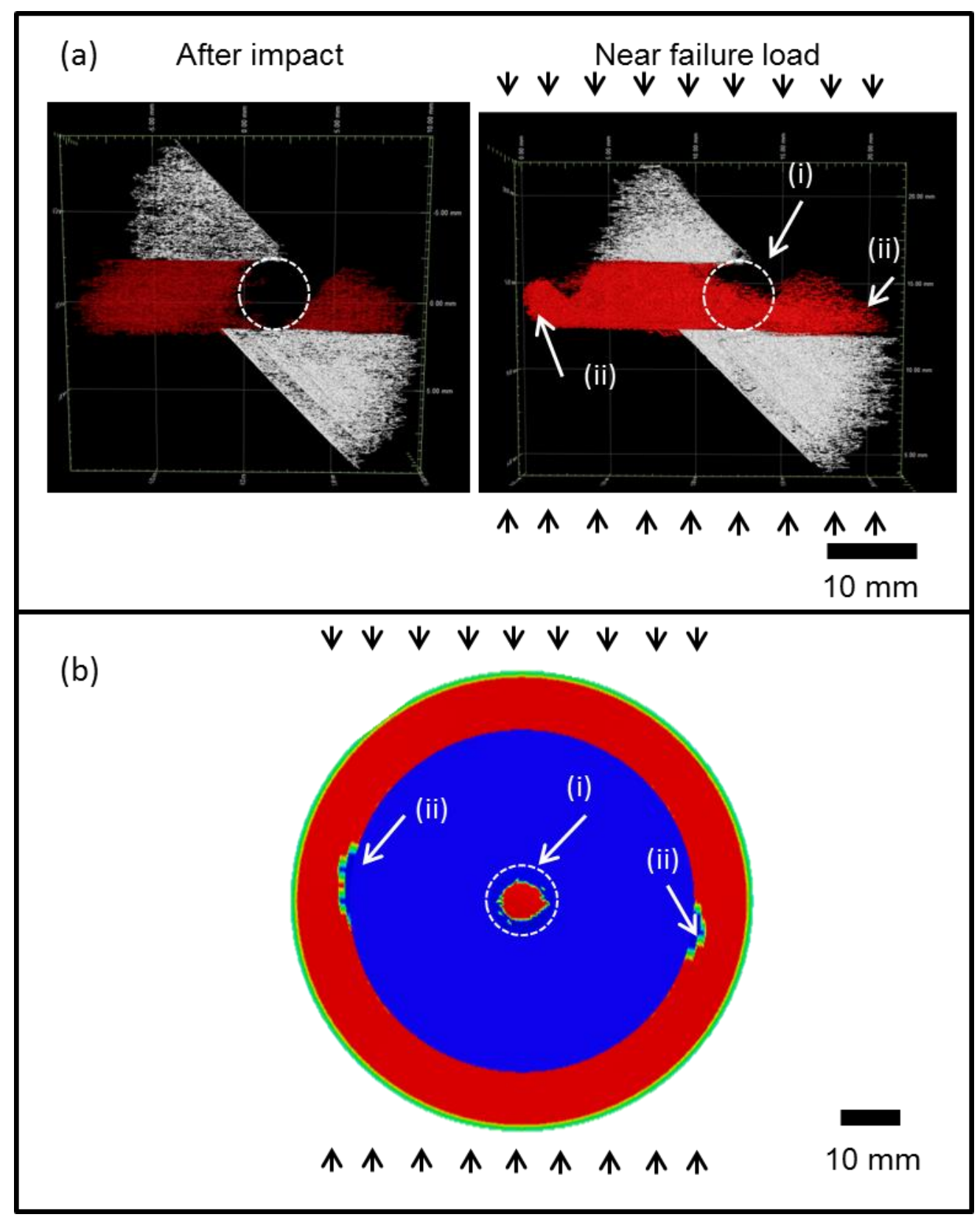

Figure 13: Comparison between (a) experimental X-ray CT scans showing delaminations (red and white) and (b) FE delaminations (blue). (i) White dashed circle indicated undamaged cone region with delamination growth into this region. (ii) Delamination growth on the outer edges of the damage area 
(a)

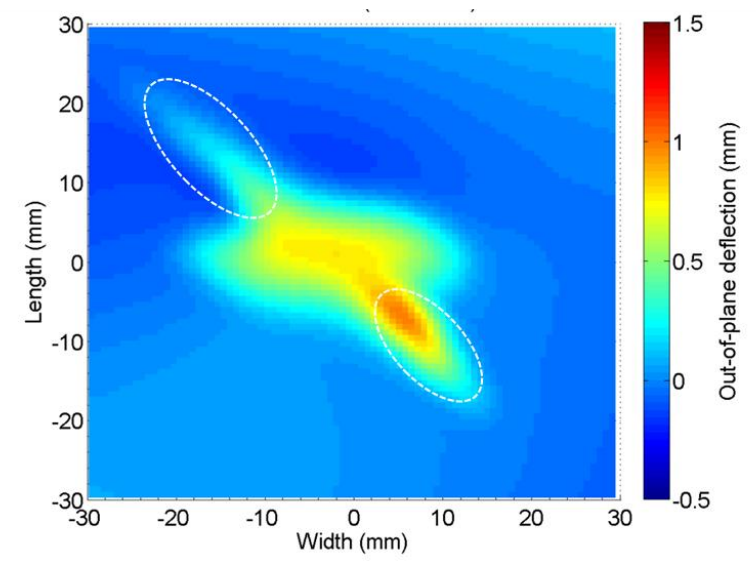

(b)

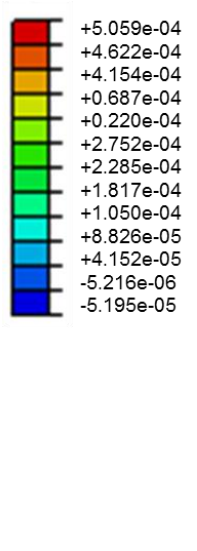

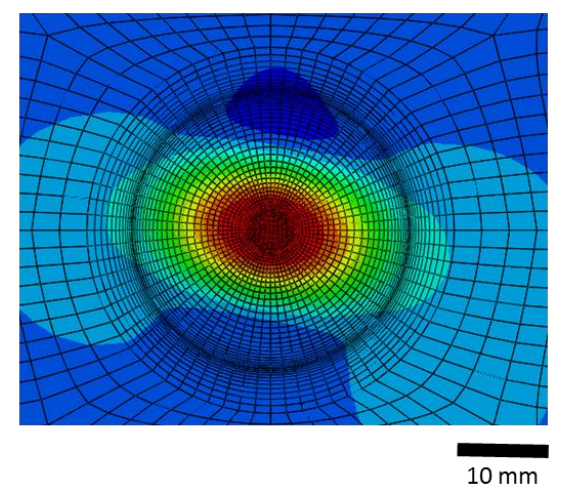

Figure 14: 3D buckling behaviour on the non-impacted side of the coupon for (a) experimental determined using stereo DIC, and (b) FE model prediction. 